\begin{tabular}{ccc}
\hline \multicolumn{3}{c}{ ANNALES } \\
UNIVERSITATIS MARIAE CURIE-SKŁODOWSKA \\
LUBLIN - POLONIA \\
VOL. LXVIII, 1-2 & SECTIO AA & 2013 \\
\hline
\end{tabular}

\title{
Influence of volume drop on surface free energy of glass
}

\author{
Diana Rymuszka ${ }^{\text {a }}$, Konrad Terpiłowski and Lucyna Hołysz \\ Department of Interfacial Phenomena, Faculty of Chemistry, \\ Maria Curie-Skłodowska University, Lublin, Poland \\ ${ }^{a} d \_r y m u s z k a @ v p . p l$
}

The aim of the research was to determine how the drop size affects the contact angle values and determine its optimal size for further contact angle measurements and comparison of the contact angle values measured for three probe liquids (water, formamide, diiodomethane) on the glass surface using the: sessile drop and tilting plate methods. Next, using the measured contact angles, the total surface free energy and its components were determined from the van Oss et al. (Lifshitz-van der Waals acid- base component, LWAB), Owens-Wendt, Neumann and contact angle hysteresis $(\mathrm{CAH})$ approaches. The studies showed, that drop size is very important for contact angle measurements and consequently, for surface free energy estimation.

Keywords: contact angle, surface free energy.

\section{INTRODUCTION}

Solid surface properties play a crucial role in many processes in nature, everyday life, agriculture and industry. One of the important properties is wettability, which depends on the surface hydrophilichydrophobic nature. The measure of solid surface wettability is the contact angle $\theta$, the one between the solid surface plane and the tangent to the drop settled on this solid surface in the three-phase contact point. Another very important parameter is the solid surface free energy which 
provides much valuable information about the surface properties of the examined material. There are many surface free energy approaches but in these studies only four of them will be discussed.

\subsection{Owens-Wendt approach}

Owens-Wendt [1] assumed that if the interface polar interactions (non-dispersive) appear, then based on Young equation [2] dispersive $\gamma_{s}^{d}$ and polar $\gamma_{s}^{p}$ components can be determined as a geometric function of these components:

$$
\gamma_{l}(1+\cos \theta)=2\left(\gamma_{s}^{d} \gamma_{l}^{d}\right)^{1 / 2}+2\left(\gamma_{s}^{p} \gamma_{l}^{p}\right)^{1 / 2}-\pi_{e}
$$

Assuming that $\pi_{e} \cong 0$ and based on the average contact angle values measured for two liquids (apolar and polar), the $\gamma_{s}^{d}$ and $\gamma_{s}^{p}$ components of solid can be calculated. This method is commonly used for surface free energy determination especially for polymers.

\section{2. van Oss, Good, Chaudhury approach}

Van Oss et al. expressed the surface free energy as a sum of two components: the Lifshitz van der Waals $\left(\gamma_{i}^{L W}\right)$ component and the acidbasic component of Lewis $\left(\gamma_{i}^{A B}\right)[3]$ :

$$
\begin{gathered}
\gamma_{i}^{L W}=\gamma^{d}+\gamma^{p}+\gamma^{i} \\
\gamma_{i}=\gamma_{i}^{L W}+\gamma_{i}^{A B}
\end{gathered}
$$

According to these authors [4], the acid-basic interactions component $\left(\gamma_{i}^{A B}\right)$ can be also expressed using the geometric average:

$$
\gamma_{i}=\gamma_{i}^{L W}+\gamma_{i}^{A B}=2\left(\gamma_{i}^{+} \cdot \gamma_{i}^{-}\right)^{\frac{1}{2}}
$$

Based on this model, adhesion work can be expressed with the components:

$$
W_{A}=\gamma_{l}(1+\cos \theta)=2 \sqrt{\gamma_{s}^{L W} \gamma_{l}^{L W}}+2 \sqrt{\gamma_{s}^{+} \gamma_{l}^{-}}+2 \sqrt{\gamma_{s}^{-} \gamma_{l}^{+}}
$$

The $\gamma_{s}^{L W}$ component and $\gamma_{s}^{+}, \gamma_{s}^{-}$parameters of the surface free energy can be determined from equation (5) by measuring the contact 
angles of three different polarity liquids whose surface tension components are known: $\gamma_{l}^{L W}, \gamma_{l}^{+}$and $\gamma_{l}^{-}$. Solving three equations with three unknowns $\left(\gamma_{s}^{L W}, \gamma_{s}^{+}, \gamma_{s}^{-}\right)$allows determination of the surface free energy components $\left(\gamma^{L W}, \gamma^{A B}\right)$ and finally the total surface free energy value.

\subsection{Neumann approach}

In contrast to the surface free energy estimation methods based on its separation into the independent components, where using two or three different liquids is required, in the Neumann approach only one liquid is desirable [5]:

$$
\left(\gamma_{s} / \gamma_{l}\right)^{0.5} \exp \left[-\beta_{1}\left(\gamma_{l}-\gamma_{s}\right)^{2}\right]=0.5(1+\cos \theta)
$$

\subsection{Contact angle hysteresis}

Chibowski proposed a quantitative interpretation of the contact angle hysteresis $(\mathrm{CAH})[6,7]$. He related the total surface free energy of solid $\left(\gamma_{s}{ }^{t o t}\right)$ with the three measurable parameters: advancing $\left(\theta_{a}\right)$ and receding $\left(\theta_{r}\right)$ contact angles and liquid surface tension $\left(\gamma_{L}\right)$ used during measurements. The surface free energy estimated in this way depends on the interaction type and its size occurring at the interface.

$$
\gamma_{s}^{t o t}=\frac{\gamma_{1}\left(1+\cos \theta_{\mathrm{a}}\right)^{2}}{\left(2+\cos \theta_{r}+\cos \theta_{\mathrm{a}}\right)}
$$

\section{MATERIALS AND METHODS}

\subsection{Materials}

$20 \mathrm{~mm}$ x $30 \mathrm{~mm}$ glass plates (Comex, Wrocław, Poland) which were cut from microscope slides were used during the studies. Before measurements the plates were washed with water containing detergent, and then rinsed with the Milli-Q 185 system water several times. The next step included plates washing in methanol and doubly-distilled water in the ultrasonic for 15 minutes and then dried in a dryer. Before contact angle measurements all plates were stored in a desiccator. 
The liquids used during the contact angle measurements: diiodomethane (99\%, Aldrich, Germany), formamide, (98\%, Aldrich, Germany, deionizated water Milli-Q $185(\mathrm{pH} \approx 6.5)$.

The liquids used during the glass plates preparation: methanol, (POCH S.A, Poland), redestillated water.

\subsection{Methods}

Contact angle measurements using the sessile drop method

Digidrop GBX Contact Angle Meter (France) equipped with the video-camera system and computer software was used for the contact angle measurements by the sessile drop and tilting plate methods. In the sessile drop method, the advancing contact angles of water and two other probe liquids, diiodomethane and formamide of $1,2,3,4,5,6,7,8 \mu 1$ volume were measured after settling droplets with appropriate drop volume on the glass surface. Then after sucking of $1 / 3$ from the droplet into the syringe, the receding contact angle was measured. The contact angles of probe liquids were measured at $20 \pm 1^{\circ} \mathrm{C}$ in a closed chamber.

Contact angle measurements using the tilting plate method

Using the tilting plate method the contact angles were measured on the glass surface which is inclined relative to the optical axis and in that position liquid gathered on one side of the droplet and retracted on the other one. The droplet was set in the chamber in front of the Contact Angle Apparatus camera and then using a small table, droplet was tilted at the appropriate angle. The whole process was filmed. Then the frame of a movie piece in which the droplet was the most deformed but did not slide from the surface was chosen and the advancing (in front of droplet) and receding (rear drops) contact angle were measured using the "Manual mode 1" method.

\section{Surface free energy estimation}

The total surface free energy and its components were determined based on the measured contact angles for three probe liquids (water, formamide, diiodomethane) from van Oss et al. (Lifshitz-van der Waals acid- base component, LWAB), Owens-Wendt, Neumann and contact angle hysteresis $(\mathrm{CAH})$ approaches. 


\section{Surface roughness}

The surface mapping studies was carried out by AFM method using a standard silicon tip in the "contact mode", at room temperature in an open system. The results were processed with the program WSxM 4.0, Develop 8.0.

\section{RESULTS AND DISCUSSION}

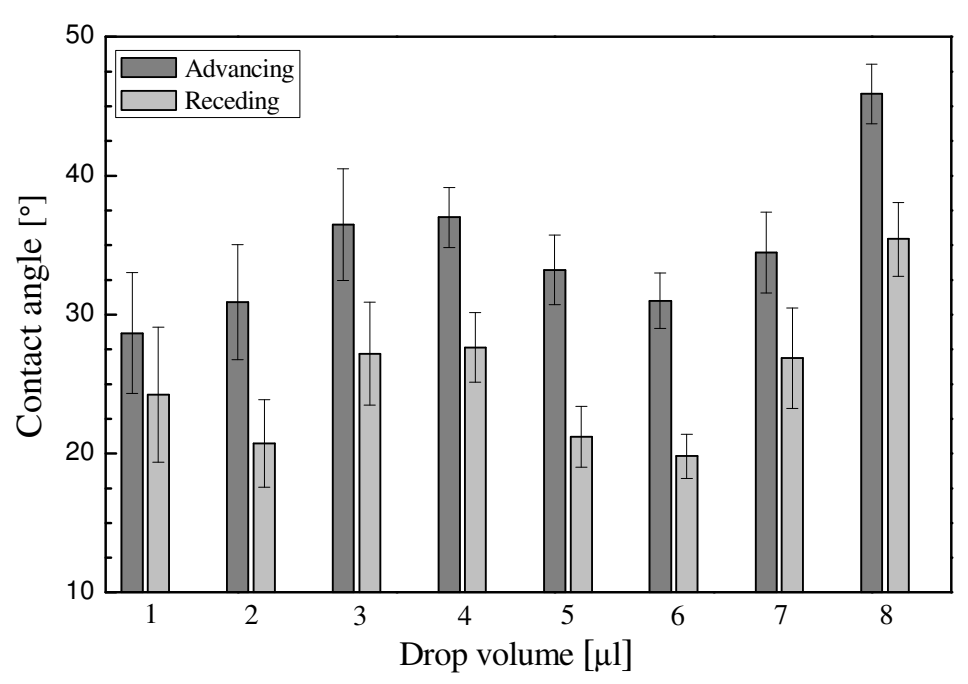

Fig. 1. Values of advancing and receding contact angles measured for water on the glass surface.

As is it shown in Fig. 1 in a range of values, the water contact angles depend on the droplet volume used for measurements. The contact angle of $1 \mathrm{~cm}^{3}$ droplet was the smallest volume with the biggest standard deviation. The surface covered with the drop during measurements was about $0.6 \mathrm{~mm}^{2}$. However, the average roughness on the glass surface determined by the AFM technique was $2.6 \mathrm{~nm}$ (Fig. 3). Large deviation shows that the droplets with that volume are very sensitive to the local surface irregularities. Therefore using such small droplets is not appropriate because they are not representative even with such small surface roughness. It seems that the larger volume of droplets $\left(2-4 \mathrm{~cm}^{3}\right)$ is not so sensitive to the glass surface irregularities but the standard deviation is still about twice larger than that for droplets with a slightly larger volume. The water contact angles measured on the glass surface in the range of $5-7 \mathrm{~cm}^{3}$ are much more representative because the surface 
area occupied by the droplets is larger. They are not so sensitive to the local surface irregularities, as indicated by the standard deviation. However, for $7 \mathrm{~cm}^{3}$ drop volume and larger, gravity has much bigger influence on the contact angle measurements (Fig. 2). The standard deviation of droplets volume from 2 to $8 \mathrm{~cm}^{3}$ does not exceed $1.4^{\circ}$.

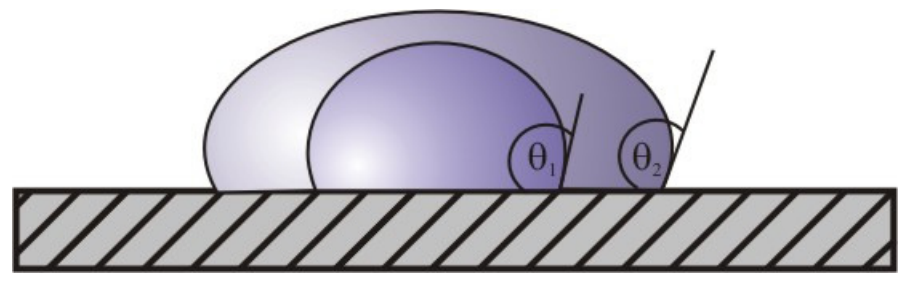

Fig. 2. Liquid droplets behaviour on the solid surface depending on the droplet volume.

As shown in Fig. 2, the contact angle $\theta_{2}$ is a little larger than $\theta_{1}$. Although, using droplets with much larger volume causes that they occupy much bigger surface and are less sensitive to the local heterogeneity which leads to the gravity influence exposure. Based on the obtained results (Fig. 1) $6 \mathrm{~cm}^{3}$ droplets seem to be optimal for the contact angle measurements.

Very similar studies were carried out by Good and Koo [8]. They investigated the droplet volume influence on the advancing and receding contact angles. As the experimental materials teflon and poly(methyl methacrylate) were used and as the tested liquids: water, ethyl glycol and decane. The contact angles were measured for a different droplet diameters $0.1-1.2 \mathrm{~cm}$. The studies show that the contact angle can increase with diameter droplet increasing to reach a certain threshold value typical of the solid-liquid system. This increase is particularly pronounced in the case of water droplets deposited on the teflon. The contact angle rises by $15^{\circ}$ when the droplet diameter increases in the $0.1-0.4 \mathrm{~cm}$ range. The authors explained this effect with as due to the existence hydrophobic areas on the teflon surface.

In the process of his studies including the effect of droplet size on the contact angle and using various solid surfaces Drelich [9], found that advancing contact angles in many real systems, namely smooth homogeneous, heterogeneous, slightly heterogeneous and other surfaces, were practically constant until the $7 \mathrm{~mm}$ water droplets diameter was achieved. 
During the studies of solid surfaces covered with a wax layerobserved contact angle changes with the droplet volume changes [10]. The contact angles changed from $50^{\circ}$ to $90^{\circ}$ if the droplet volume was $0.4-3.65 \mathrm{~cm}^{3}$. For small droplet $\left(0.1 \mathrm{~cm}^{3}-0.4 \mathrm{~cm}^{3}\right)$ the contact angle was almost constant at $88^{\circ}-90^{\circ}$. Mack, like other researchers, attributed this relationship to the result of the gravity presence. In light of the above studies it is difficult to agree with the results of the research presented by Mack. In order to eliminate the gravity effect, the droplets volume should not exceed $0.5 \mathrm{~cm}^{3}$. As was it shown earlier, the droplets are very sensitive to the local surface heterogeneity.

$\mathrm{He}$, Neelesh and Patankar studied out the drop volume influence on the contact angle on rough surfaces [11]. Water contact angles were measured on the rough polydimethylsiloxane surface modified with plasma. The water droplets volume on the surface changed from 1 to $8 \mathrm{~cm}^{3}$. The aim of the study was to compare the droplets volume effect on the measured contact angle in two states: when the liquid wets the surface completely (Wenzel state) and when the ruggedness does not allow for the complete surface wetting (Cassie-Baxter state). In all described cases the contact angle increases with the increasing drop volume. This is much clearer for the droplets whose behaviour on the surface can be explained by the mechanism proposed by Wenzel. Confirmation of the hypothesis that gravity does not affect smaller droplets can be found by computer simulations carried out by Vafei and Podowski [12].

Considering the theoretical issue of the droplet size effect on the contact angle Iwamatsu [13] modified the Cassie equation [14]. He assumed the cylindricity droplet shape and considered chemically heterogeneous smooth solid surface. He concluded that the contact angle is a linear volume function. The line slope is different and depends on the surface hydrophobicity on which the contact angle was measured. In the light of the research carried out in this paper, the calculations presented by Iwamatsu only for the $1-3 \mu \mathrm{m}$ volume range can be confirmed. 
a)

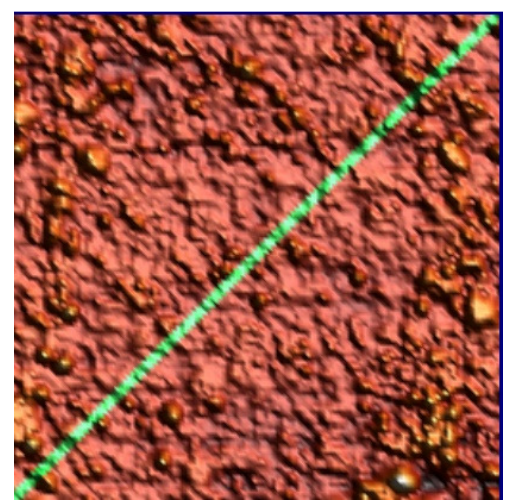

b)

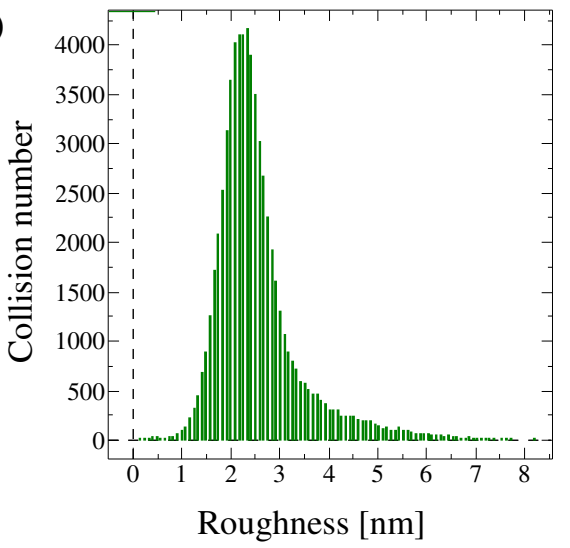

$R_{r m s}=0.94 \mathrm{~nm}$, Av. Heigh $=2.6 \mathrm{~nm}$

c)

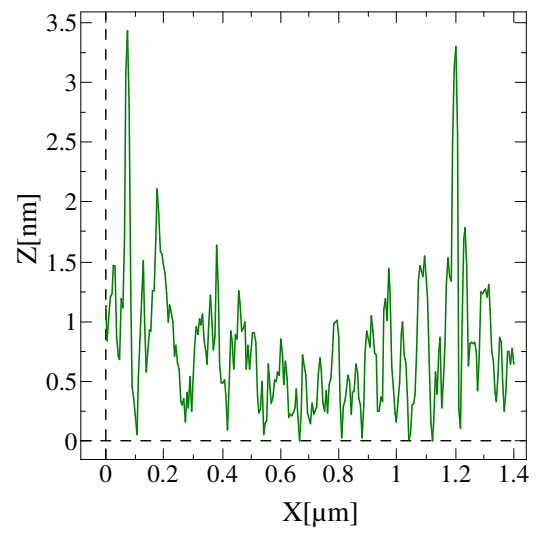

Fig. 3. a) 3D AFM glass mapping (1 $1 \mu \mathrm{m} \times 1 \mu \mathrm{m})$; b) Roughness distribution of the examined surfaces and $R_{r m s}$ and average roughness; c) Surface profile along the line shown in 3D imaging. 
The glass surface shown in Figure 3 is characterized by roughness distribution relocated toward higher values. The average roughness is $0.94 \mathrm{~nm}$ and $R_{r m s}=2.6 \mathrm{~nm}$. The surface profile shows two distinct spikes in the roughness distribution.

Detailed description of the topographical nature of the surface roughness is important because the volume of liquid particles used for the contact angle measurements is significantly different: for diiodomethane $134 \AA^{3}$, water $30 \AA^{3}$ and formamide $66 \AA^{3}$. Therefore, water can more easily penetrate into the surface grooves than the other liquids. However, liquid behaviour on the solid surfaces depends also on its surface tension and polar interactions on the solid.

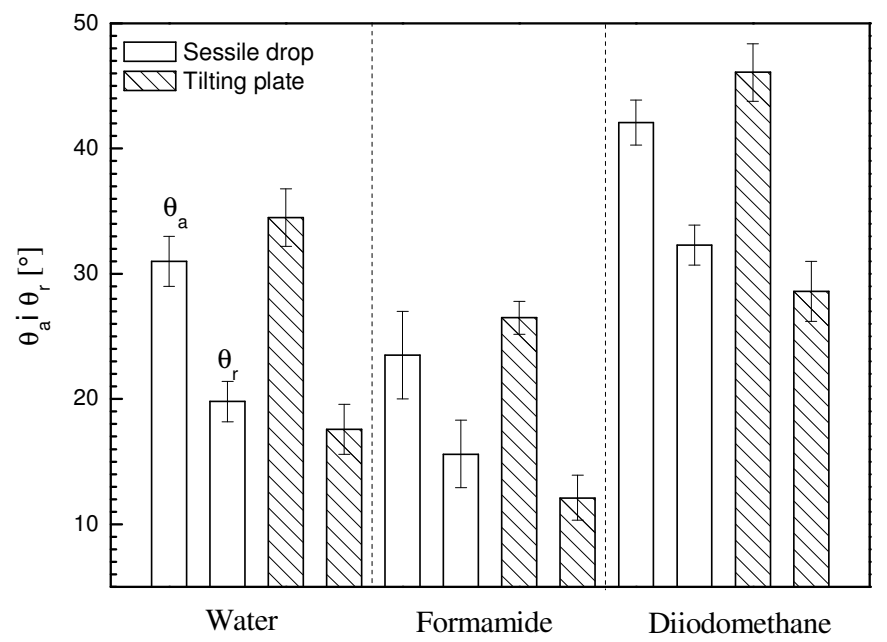

Fig. 4. Advancing $\left(\theta_{\mathrm{a}}\right)$ and receding $\left(\theta_{\mathrm{r}}\right)$ contact angles of probe liquids measured on the glass surface using the sessile drop (empty columns) and tilting plate methods (hatched columns).

Advancing and receding contact angles of probe liquids measured by both techniques are shown in Fig. 4. The advancing contact angles measured using the tilting plate method are larger by several degrees in comparison to the contact angles measured by the sessile drop method. The receding contact angles measured using the tilting plate method are mostly lower than those measured using the sessile drop method. The diiodomethane contact angle hysteresis shows large differences between two methods used for contact angle measurements. This is an apolar liquid which interacts with the solid surface practically only by dispersion forces $\gamma_{l} \approx \gamma_{l}^{d}=50.8 \mathrm{~mJ} / \mathrm{m}^{2}$, and for water $\gamma_{l}^{d}=21.8 \mathrm{~mJ} / \mathrm{m}^{2}$, formamide 
$\gamma_{l}^{d}=39.0 \mathrm{~mJ} / \mathrm{m}^{2}$ [15]. Therefore it can be concluded that dispersive interactions are primarily responsible for the differences in the values of hysteresis. Furthermore, a diiodomethane molecule has four times larger volume in comparison to the water molecule and twice to formamide. It can be explained by small diiodomethane hysteresis using the mechanism proposed by Cassie Baster, assuming air-filled voids existence under the droplet. Based on the measured contact angles of probe liquids, it can be seen that there is no simple relationship between the surface roughness and the surface tension of the liquid and its contact angle.

Table 1. Contact angle hysteresis values measured on the glass plates using the sessile drop and tilting plate methods.

\begin{tabular}{rccc}
\hline \multirow{2}{*}{ Method } & \multicolumn{3}{c}{ Liquids } \\
\cline { 2 - 4 } & water & formamide & diiodomethane \\
\hline Sessile drop & $11.2 \pm 1.8$ & $7.9 \pm 3.1$ & $9.8 \pm 1.7$ \\
\hline Tilting plate & $16.9 \pm 2.2$ & $14.4 \pm 1.5$ & $17.5 \pm 2.4$ \\
\hline
\end{tabular}

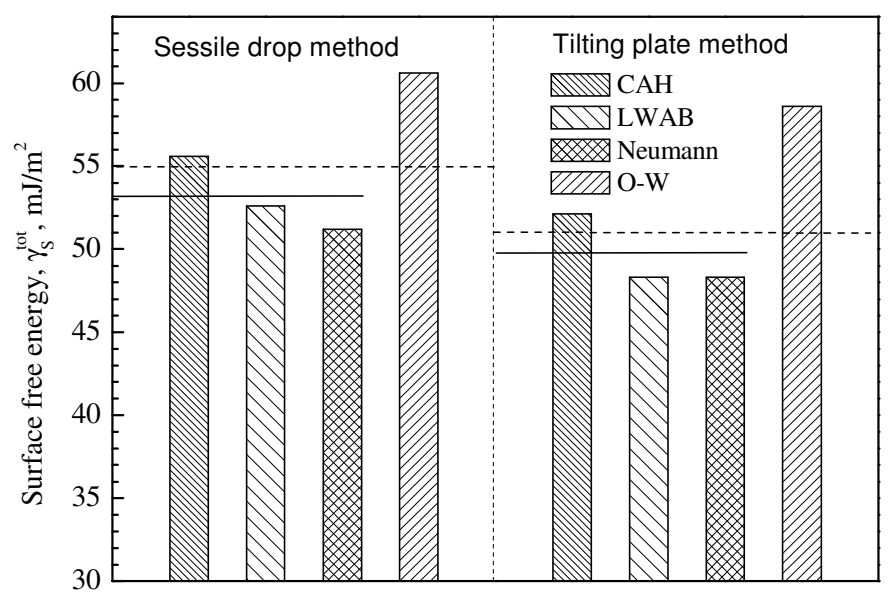

Fig. 5. Surface free energy calculated from different theoretical approaches to the glass surface.

Based on the contact angles measured using both methods shown in Fig. 4 the surface free energy was calculated using the theoretical approaches: contact angle hysteresis (CAH), van Oss, Good, Chaudchury (LWAB), Neumann and Owens-Wendt approaches. The results of surface 
free energy estimation are presented in Fig. 5. The dashed horizontal line in the figure indicates the arithmetic average of all used theoretical approaches. However, the Owens - Wendt approach gives the highest surface free energy value, so the horizontal continuous line indicated the arithmetic average of the three approaches: contact angle hysteresis $(\mathrm{CAH})$, van Oss, Good Chaudchury (LWAB) and Neumann. The surface free energy calculated from the contact angle hysteresis is the arithmetic average of the energy calculated for the three probe liquids: water, diiodomethane, formamide. The highest surface free energy values were observed for the surface free energy calculated using the Owens - Wendt and the $\mathrm{CAH}$ approaches. Higher contact angle hysteresis values are observed for the contact angles measured using the tilting plate method. Not much higher surface free energy values are observed from the estimation based on the contact angles measured using the sessile drop method but both contact angle measurement techniques: sessile drop and tilting plate, can be widely used for energy calculations from the contact angle hysteresis.

\section{CONCLUSIONS}

The contact angle of $1 \mathrm{~cm}^{3}$ droplet was the smallest volume with the biggest standard deviation. The water contact angles measured on the glass surface in the range of $5-7 \mathrm{~cm}^{3}$ are much more representative because the surface area occupied by the droplets is higher. They are not so sensitive to the local surface irregularities. For a $7 \mathrm{~cm}^{3}$ drop and larger, gravity has much bigger influence on the contact angle measurements. Based on the obtained results $6 \mathrm{~cm}^{3}$ droplet volume seems to be the optimal for the contact angle measurements. The advancing contact angles measured using the tilting plate method are larger by several degrees in comparison to the contact angles measured by the sessile drop method. On the other hand, the receding contact angles measured using the tilting plate method are mostly lower than those measured using the sessile drop method. Furthermore, the largest surface free energy values were calculated using the Owens-Wendt and the $\mathrm{CAH}$ approaches. The surface free energy estimated using the contact angles measured by means of the sessile drop method is $4 \mathrm{~mJ} / \mathrm{m}^{2}$ larger. 


\section{REFERENCES}

[1] D. K. Owens, R. C. Wendt, J. Appl. Polymer. Sci., 13, 1741, (1969).

[2] T. Young, Philos. Trans. R. Soc. London, 95, 65, (1895).

[3] C. J. van Oss, R. J. Good, M. K. Chaudhury, Langmuir, 4, 884-891, (1988).

[4] R. J. Good, M. K. Chaudhury, Plenum Press, New York, 153, (1991).

[5] M. Żenkiewicz, POLIMERY 52, no. 10, 764 (2007).

[6] E. Chibowski, VSP, Utrecht 2002, Netherlands.

[7] E. Chibowski, Advances in Colloid and Interface Science, 103, 149-172, (2003).

[8] R. J. Good, M. N. Koo, J. Colloid Interface Sci., 71, 2, 283, (1979).

[9] J. Drelich, J. Adhesion, 63, 31, (1997).

[10] G. L. Mack, J. Phys. Chem., 40, 159, (1936).

[11] B. He, J. Lee, N. A. Patankar, Colloids and Surf. A., 248, 101, (2004).

[12] S. Vafei, M. Z. Podowski, Nuclear Engineering and Design, 235, 1293, (2005).

[13] M. Iwamatsu, J. Colloid Interface Sci., 297, 772, (2006).

[14] A. B. D. Cassie, Discuss. Faraday Soc., 3, 11, (1948).

[15] W. Mizerski, Tablice chemiczne, Adamant, Warszawa, 2008.

\section{CURRICULUM VITAE}

Diana Rymuszka. PhD student of Maria Curie-Skłodowska University in Lublin, Poland, Department of Interfacial Phenomena, Faculty of Chemistry.

Konrad Terpiłowski. Assistant Professor, Department of Interfacial Phenomena, Faculty of Chemistry, Maria Curie Skłodowska University in Lublin, Poland. Author of several dozen publications. 